\title{
Viabilidad económica de las cooperativas vecinales para el envejecimiento activo. Málaga como caso de estudio
}

\author{
Carlos Rosa-Jiménez ${ }^{1}$ | Nuria Nebot ${ }^{2}$ \\ Recibido: 08-12-2020 | en su versión final: 15-02-2021
}

Resumen

\begin{abstract}
El envejecimiento progresivo de la población mundial es un fenómeno que preocupa a instituciones y organismos nacionales e internacionales. El envejecimiento en el lugar supone una tendencia deseada por las personas mayores que han vivido mucho tiempo en una comunidad; sin embargo, cuenta con cuatros grandes problemas de este sector de la población: la soledad, la obsolescencia de las viviendas, la falta de recursos ante la incertidumbre económica de las pensiones, y el impacto de la pandemia del COVID-19. Este artículo pretende avanzar en las investigaciones sobre cooperativismo de personas mayores, a partir del concepto de cooperativa vecinal, entendida como una empresa social donde participan todos los vecinos de una barriada con el fin de dotar a sus socios de todas las prestaciones de habitabilidad con una aportación económica muy reducida. Esta investigación analiza su viabilidad económica en el sector específico de las personas mayores, al permitir obtener las prestaciones específicas de este sector de población (incluida la mejora de la vivienda y resolver los problemas de soledad obligada) y gestionar además un parque social de alquiler, en unos momentos de dificultad del acceso a la vivienda de alquiler. Se propone una metodología basado en el estudio económico de una implantación teórica en un caso de estudio en Málaga. El estudio concluye analizando la viabilidad económica y el incremento en las prestaciones sociales que permite su implantación, gracias a la contratación de auxiliares de apoyo, en la oferta de alquiler social a precio tasado.
\end{abstract}

Palabras clave: envejecimiento; soledad; vivienda de alquiler; apoyo mutuo

Citación Rosa-Jiménez, C y Nebot, N. (2021). Viabilidad económica de las cooperativas vecinales para el envejecimiento activo. Málaga como caso de estudio. ACE: Architecture, city and Environment, 15(45), 10368. DOI: http://dx.doi.org/10.5821/ace.15.45.10368

\section{Economic Viability of Neighborhood Cooperatives for Active Aging. Malaga as a Case Study}

\begin{abstract}
The progressive aging of the world population is a phenomenon that worries national and international institutions and organizations. Aging in the place is a trend desired by older people who have lived a long time in a community, however, it has four major problems in this sector of the population: loneliness, obsolescence of housing, lack of resources given the economic uncertainty of pensions, and the impact of the COVID-19 pandemic. This article aims to advance research on cooperativism of older people, based on the concept of neighborhood cooperative, understood as a social enterprise where all the residents of a neighborhood participate to provide their members with all the habitability benefits with a low economic contribution. This research analyzes its economic viability in the specific sector of the elderly, by making it possible to obtain the specific benefits of this sector of the population (including the improvement of housing and solving the problems of forced solitude), also managing a social rental park, in moments of difficult access to rental housing. A methodology based on the economic study of a theoretical implementation in a case study in Malaga is proposed. The study concludes by analyzing the economic viability and the increase in social benefits that allows its implementation, thanks to the hiring of support assistants, in the offer of social rent at an appraised price.
\end{abstract}

Keywords: aging; loneliness; rental housing; mutual support

1 Doctor Arquitecto. Subdirector del Instituto Interuniversitario UPC-UMA Hábitat, Turismo y Territorio (ORCID: 0000-0001-6356-8734; Scopus Author ID: 56233277900), Doctora arquitecta. Instituto Interuniversitario UPC-UMA Hábitat, Turismo y Territorio (ORCID: 0000-0001-6187-0972; Scopus Author ID: 57192719062). Correo de contacto: çrosa@uma.es 


\section{Introducción. El problema del envejecimiento de la población y la falta de alquileres}

La cooperativa es uno de los mejores exponentes de la economía social, dado su carácter no lucrativo y compromiso social derivado de su modo de gobernanza. Es considerado por la Unión Europa como un agente de salida estructural de la crisis económica por su capacidad de regular los numerosos desequilibrios del mercado laboral (desempleo, inestabilidad laboral, exclusión social, etc.) y su capacidad de movilizar el potencial económico endógeno, construyendo colectivamente nuevas sinergias para la revitalización de las zonas locales (Monzón y Chaves, 2012). Efectivamente, la Estrategia Europa 2020 establece tres prioridades básicas: crecimiento inteligente, crecimiento sostenible y crecimiento integrador. Las cooperativas han demostrado que contribuyen al desarrollo de una economía basada en el conocimiento y la innovación (crecimiento inteligente); cuentan con sistemas de valores que materializan la solidaridad con el entorno, internalizando los costes sociales y generando externalidades positivas (crecimiento sostenible); y, en el ámbito de la lucha contra la pobreza y la exclusión social, el auge de las empresas sociales ha sido considerable (crecimiento integrador). Además, la capacidad de las cooperativas para resolver las nuevas necesidades sociales han revitalizado su importancia (Monzón y Chaves, 2012).

Las cooperativas vecinales son un modelo de gestión de regeneración urbana basada en la creación de una cooperativa de consumo y trabajo para la prestación de servicios de habitabilidad para una barriada o un conjunto de ellas, donde participan todos los residentes (propietarios o no) así como los comercios locales que allí residen (Rosa-Jiménez, Márquez-Ballesteros, y Navas-Carrillo, 2017). Su adaptación al sector poblacional de las personas mayores puede hacer frente a dos graves problemas actuales: 1) el incremento constante de personas mayores que viven en soledad y 2) la escasez de vivienda en régimen de alquiler. Dos aspectos que la creación de una cooperativa vecinal permite resolver de forma conjunta, al relacionar ambos procesos.

En relación a la primera cuestión, en España, el proceso de envejecimiento de la población se inició más tarde que en los países del norte de Europa, pero la intensidad fue mayor que en otros países vecinos (Fernández Muñoz, 2015). En 2013, la población de adultos mayores censados en 1981 se había duplicado, y para 2066 se estima que habrá 14 millones de personas mayores de 65 años, lo que representará el 34,6\% del total de 41.068 .643 residentes (Abellán, Ayala, y Pujol, 2017).

La preferencia de las personas mayores, que han vivido en comunidad durante mucho tiempo, a envejecer y residir en sus barrios se mantiene mientras dure sus redes sociales tradicionales y comunitarias (Hui, Wong, Chung, y Lau, 2014). Sin embargo, actualmente existen tres grandes problemas de este sector de la población:

a) El problema de la soledad. En relación al aislamiento social, en 2016, 75 millones de ciudadanos europeos informaron estar socialmente aislados. En 2017, el 18\% o casi uno de cada cinco ciudadanos de la UE de 65 años o más estaba en riesgo de pobreza o exclusión social (European Commission, 2019). Existe un creciente reconocimiento para promover la inclusión social de las personas mayores como parte de las políticas nacionales y de la UE (European Commission Joint Research Centre, 2018), dado que las personas con privaciones materiales y menores ingresos tienen menos probabilidades de participar en actividades sociales. Las personas mayores que viven solas lo hacen por el deseo y la satisfacción de continuar en sus viviendas y en su entorno social conocido, dado el alto valor afectivo que los mayores otorgan a la casa, plagada de recuerdos de los que no quieren desprenderse (López, 2005). En el contexto español, el estudio de Costa-Font, Elvira, y Mascarilla-Miró (2009) reveló que el 78\% de las personas mayores

ACE, 15 (4.5) CC BY-ND 3.0 ES | UPC Barcelona, España | Viabilidad económica de las cooperativas vecinales para 
prefieren permanecer en sus vivienda, datos que coinciden con estudios internacionales como los de Borgloh y Westerheide (2012).

b) Las necesidades especiales de habitabilidad. Sin embargo, envejecer en el lugar, supone residir en viviendas sobredimensionadas para sus necesidades y, en muchos casos, no adaptadas. En la ciudad esto puede suponer un abandono ya que muchas personas mayores, al quedar viudas (especialmente las mujeres), venden su casa para adquirir otra vivienda de tamaño más reducido y con mejores equipamientos cercana a casa de sus hijos (López, 2005, p. 89). Por el contrario, las personas mayores más jóvenes y las personas con movilidad reducida son más proclives a la adaptación de sus viviendas; sin embargo, no todas las personas mayores cuentan con recursos suficientes para poder afrontar una reforma integral.

c) La incertidumbre económica de las pensiones. El envejecimiento de la población se traduce en una reducción del número de ocupados por pensionista, lo que está obligando a modificar el sistema de pensiones para que sea sostenible financieramente. Ya en 2003, Zuribi (2003) propuso como medidas una reducción del coste mediante el retraso de la edad de jubilación, y el aumento de ingresos mediante la subida de impuestos, cotizaciones o la creación de un fondo de reserva. La reforma del sistema público de pensiones de 2013 ya ha retrasado paulatinamente la edad de jubilación de los 65 a los 67 en el 2027 (Herce, 2016). Aun así, se plantea la necesidad de una capitalización complementaria, ya que los escenarios futuros son pesimistas dado el acelerado deterioro financiero del sistema de pensiones. Por ello es preciso buscar modelos que busquen la equidad en términos de distribución del consumo y de paliar la pobreza de la vejez, donde es prioritario aumentar la financiación del sistema sin aumentar los tipos de cotización (Ayuso, Guillén, y Valero, 2013). De acuerdo con Herce (2016) la creciente esperanza de vida supone un reto de sostenibilidad y suficiencia para las pensiones, públicas o privadas, de capitalización o reparto, que obligará a ir más allá de las importantes reformas adoptadas en los últimos años.

A los retos del envejecimiento en el lugar, debemos añadir la dificultad al acceso a la vivienda de alquiler que sufren las grandes ciudades españolas, agravada por la actividad turística. Por ejemplo, en Málaga capital, según registros del Instituto Municipal de la Vivienda' existían en 2016 aproximadamente 15.000 familias de jóvenes menores de 35 años sin posibilidad de acceso a una la vivienda. El auge de los alquileres vacacionales con la inclusión de la oferta privada colaborativa en el mercado turístico de empresas como AirBNB (Figura 1) o Homeaway parece incidir en el incremento del valor inmobiliario del alquiler de vivienda. El alquiler de larga estancia (destinado principalmente a residentes) sufre una importante competencia por el alquiler de corta estancia (destinado principalmente a visitantes y turistas). El crecimiento exponencial de AirBNB ha alcanzado en 2018 a más de 200.000.000 millones de huéspedes en todo el mundo (Airbnb, 2018). El auge de las viviendas turísticas ha supuesto un crecimiento acumulado del precio de la oferta del alquiler en el período 2013-19, que, en Málaga, asciende a algo más del 45\% (López Rodríguez y de los Llanos Matea, 2019).

La respuesta de la Administración al fenómeno de las viviendas de uso turístico es el desarrollo de un cuerpo normativo que intenta regularlo (Decreto 28/2016) o prohibirlo. Mientras que las administraciones con competencia en materia de vivienda tienen problemas para implementar nuevas políticas y programas que den alternativa habitacional a personas y familias con escasos recursos. Así, en el caso de Andalucía, el Plan andaluz de vivienda y rehabilitación (2016-20) no llegó a construir las 475 viviendas en régimen de alquiler accesible que proponía. Pero el objetivo de fomentar un parque de viviendas público en alquiler debe superar las dificultades financieras en la promoción de nuevas viviendas de alquiler, cuyos ritmos de ejecución son claramente insuficientes

\footnotetext{
${ }^{1}$ Según entrevista al Gerente el 12/12/2018 (Inédita).
} 
ante la demanda de vivienda. Por otro lado, las políticas de los planes de municipales de viviendas como es el caso de Málaga- han planteado alternativas de oferta pública de alquiler que han fracasado por el recelo de los propietarios².

Figura 1. Crecimiento del precio del alquiler de larga temporada en el distrito Centro de Málaga

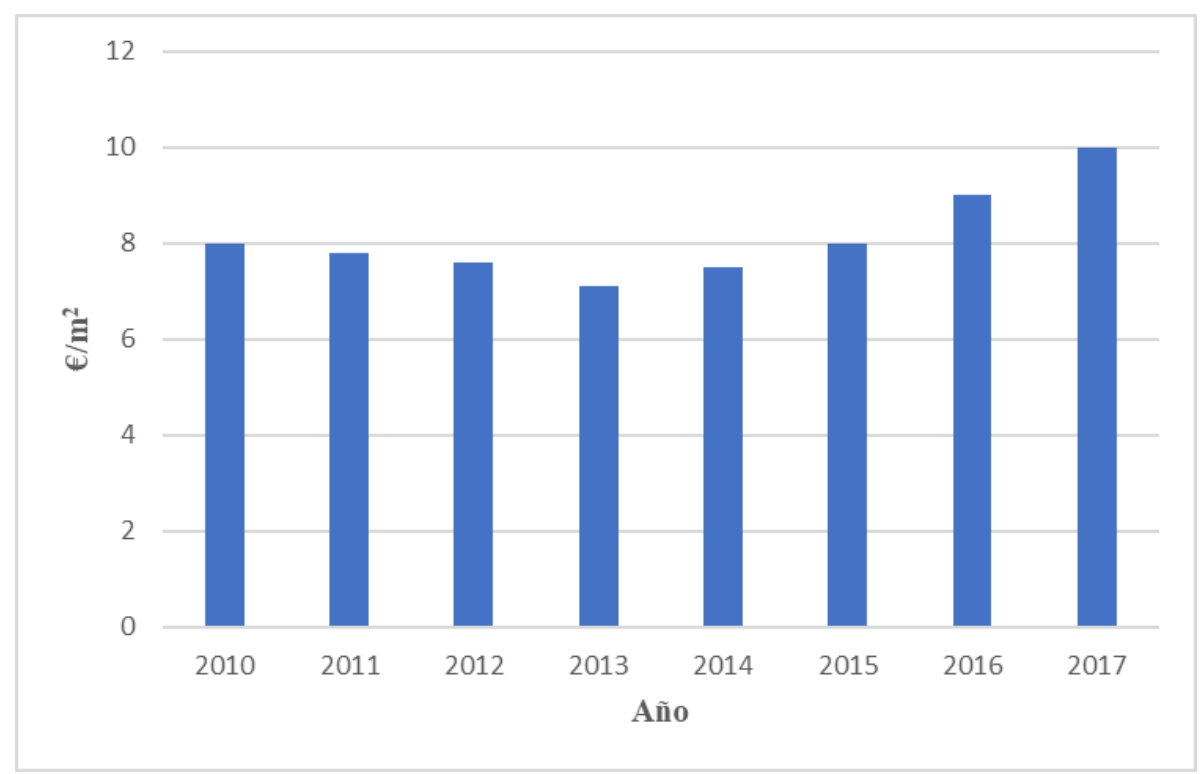

Fuente: Evolución de precio de la vivienda de alquiler (Idealista, 2019).

A tenor de los problemas planteados, este artículo demuestra que la creación de una cooperativa vecinal de personas mayores (CVPM) en una barriada es un modelo económicamente viable, y con efectos sociales positivos para este sector poblacional y para mejorar la oferta del mercado de alquiler. Para ello, se parte de una cooperativa teórica formada por socios mayores de 65 años que viven en soledad forzada y que están dispuestos a compartir la propiedad de sus viviendas en dos alternativas: 1) Viviendas de la cooperativa destinadas al agrupamiento de cooperativistas, y 2) viviendas liberadas que se ofrecen en alquiler de larga temporadas y gestionadas por la propia CVPM.

De esta forma, las personas mayores podrían adaptar aquellas viviendas que estuvieran mejor localizadas para sus necesidades, mientras que el alquiler de las viviendas restantes permitirían la obtención de recursos económicos que permitan la capitalización de los recursos económicos de las personas mayores mediante la economía social, como una forma de obtener ingresos pero también como una herramienta que pueden utilizar las administraciones para intervenir el mercado de vivienda de alquiler, especialmente para aquellas familias con peligro de exclusión social.

El modelo teórico de cooperativa vecinal de personas mayores, expresada en la Figura 2, recoge estos dos procesos: el primero de agrupación de socios en una sola vivienda que se rehabilita y adapta a las personas mayores; y el segundo, el de obtención de ingresos a partir de la oferta de alquiler de larga temporada de las viviendas desocupadas. Con los ingresos obtenidos se sufragan los gastos de rehabilitación y además permite la contratación de servicios complementarios, como personal de apoyo para las personas mayores.

\footnotetext{
2 Según noticia del Sur (Juan Soto, 14/03/2017): "El plan municipal para el alquiler social fracasa por el recelo de los propietarios. Más de 400 familias de la capital con riesgo de exclusión social no encuentra una vivienda disponible pese a tener concedida una ayuda mensual de 450 euros"
}

ACE, 15 (4.5) CC BY-ND 3.0 ES | UPC Barcelona, España | Viabilidad económica de las cooperativas vecinales para el envejecimiento activo. Málaga como caso de estudio. DOI: http://dx.doi.org/10.5821/ace.15.45.10368 
Figura 2. Modelo teórico de una cooperativa vecinal de personas mayores

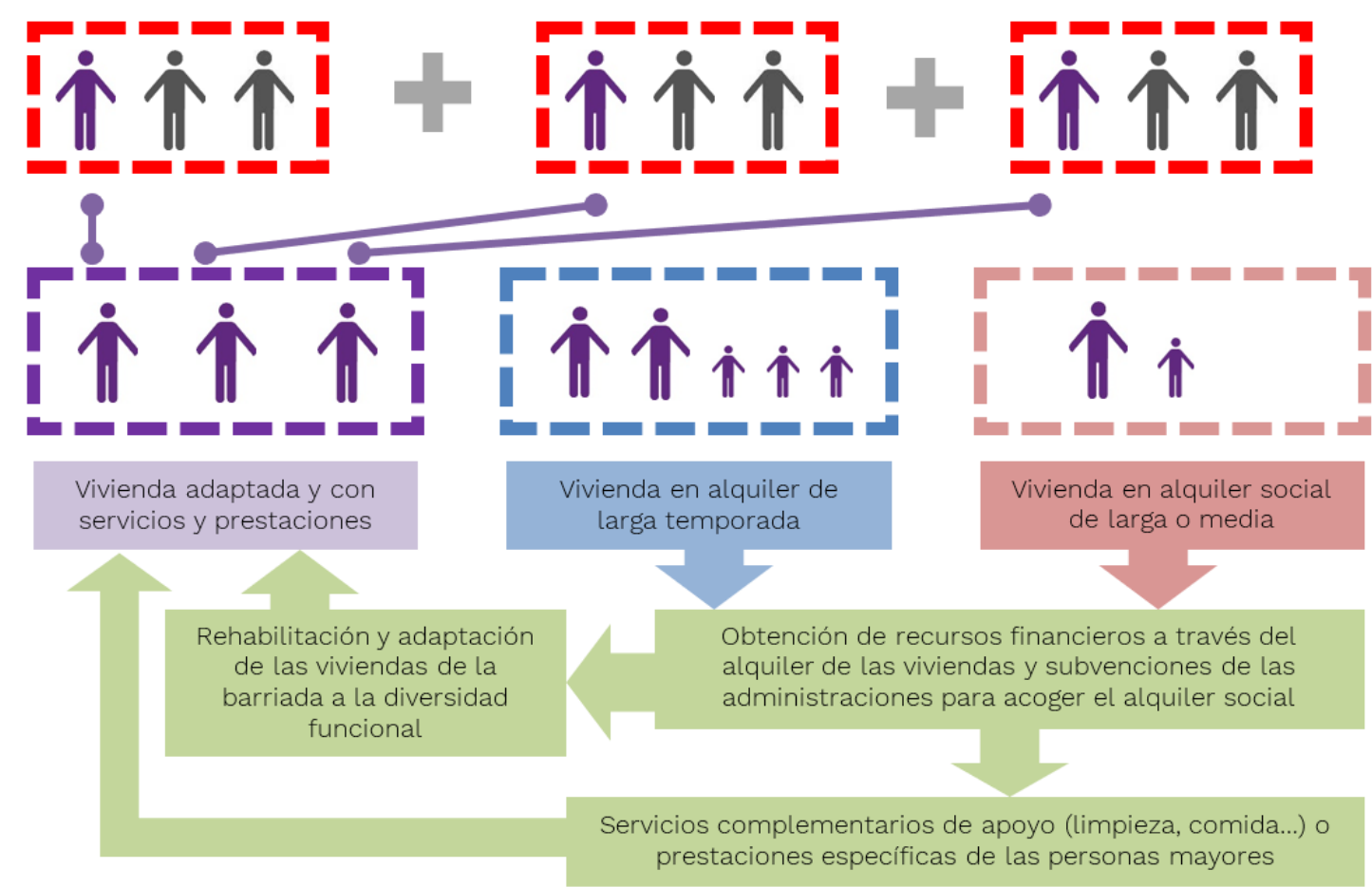

Fuente: Elaboración propia.

\section{Antecedentes. El envejecimiento activo en el lugar y el problema de la co- vivienda para personas mayores}

El envejecimiento activo se define como "el proceso de optimización de las oportunidades de salud, participación y seguridad con el fin de mejorar la calidad de vida de las personas que envejecen" (Organización Mundial de la Salud, 2002, p. 79). Los estudios sobre el envejecimiento activo desde el campo de la Arquitectura son proporcionalmente escasos en relación a otras ramas del conocimiento como la Salud y las Ciencias de la Vida, y se han desarrollado específicamente en el enfoque de las condiciones arquitectónicas de las viviendas y urbanas de la ciudad para que las barriadas sean amigables, por ello, el enfoque desde el cooperativismo constituye una aportación novedosa. Frente a la alternativa a vivir solo, el envejecimiento activo evita el desplazamiento de las residencias, la rotación periódica entre familiares o la búsqueda de parejas. En la actualidad existen dos tendencias: 1) Una primera línea que analiza el envejecimiento activo en el lugar, a través de la regeneración y reactivación de las barriadas donde han vivido las personas mayores; y 2) una segunda línea que propone el envejecimiento activo a partir de fórmulas similares a las cooperativas, pero no necesariamente en las barriadas donde han residido las personas mayores.

La primera tendencia presenta dos ventajas según Wiesel (2012): 1) Es más económico para las personas mayores que ir a centros especializados; y 2) tiene un impacto positivo en la propia regeneración urbana, frente al envejecimiento y declive de las comunidades y las limitaciones económicas de la población. Wiesel (2012) sugiere el concepto de envejecimiento positivo como recurso para el renacimiento de los barrios urbanos en dos aspectos: primero, las personas mayores disponen de más tiempo libre y pueden participar en actividades, actuando como dinamizadores

ACE, 15 (4.5) CC BY-ND 3.0 ES | UPC Barcelona, España | Viabilidad económica de las cooperativas vecinales para 
dentro de la barriada3; y segundo, mantienen la memoria colectiva de la barriada como un aspecto fundamental del entorno construido, permitiendo tanto a individuos como a la comunidad conectar con su pasado e identidad (Sandercock, 2003). En esta línea Serrano-Jiménez, Barrios-Padura, y Molina-Huelva (2018) han desarrollado protocolos de intervención en barrios con población mayoritaria de personas mayores, con el fin de garantizar las condiciones de habitabilidad en condiciones de escasa inversión pública. Sin embargo, la propuesta de los autores de una financiación avalada por la administración sólo garantiza el pago de la inversión que, finalmente, deben de asumir las personas mayores con escasos recursos.

La segunda tendencia, profundiza en el modelo de co-vivienda (co-housing) como fórmula habitacional para la promoción del envejecimiento activo, más vinculada a la fórmula del cooperativismo. En palabras de Choi (2004) una co-vivienda es un modelo de vivienda en el cual algunas personas comparten funciones importantes de la vida diaria, espacios de relación y es también una forma de "cooperativa" al ser el modelo más utilizado para su configuración. Las políticas estatales de vivienda a través del Plan estatal de la vivienda (2018-21) ya incluyen el modelo de covivienda como una alternativa habitacional para el grupo de "especial protección de los mayores de 65 años, mediante el fomento de conjuntos residenciales de viviendas con instalaciones y servicios comunes adaptados, en régimen de alquiler, de cesión en uso con renta o precio limitado" (PEV, 2018, p. 28900). Específicamente el programa de fomento de viviendas para personas mayores y personas con discapacidad facilita, además de la tradicional subvención de la accesibilidad de los edificios existentes, la creación de co-viviendas para personas mayores bien de nueva creación o rehabilitación debe "incluir instalaciones y servicios mínimos que como mínimo: asistencia social, atención médica básica veinticuatro horas disponible y cercana, limpieza y mantenimiento, dispositivos y sistemas de seguridad, restauración, actividades sociales, deportivas, de ocio y culturales, así como terapias preventivas y de rehabilitación" (PEV, 2018, p. 28905).

La co-vivienda es una opción muy positiva de proyecto de convivencia, sin embargo, existen dos problemas en la implantación de este modelo como alternativa para la soledad de las personas mayores. El primer problema es que no todas las personas mayores desean este modelo, que se relaciona más con la franja de edad comprendida entre los 50 y los 65 años (Brenton, 2008; Choi, 2004) al contar con un mayor interés en esta forma de organización. El segundo problema es que el modelo está orientado a la construcción de nuevas promociones que respondan a las necesidades del programa de convivencia que diseñen sus miembros. Aunque existen co-viviendas basadas en la rehabilitación, estas se producen en un porcentaje todavía muy reducido. Las dificultades de encontrar suelo, o un edificio completo, obligan a que la futura comunidad se desplace a la periferia de las ciudades, atraídos también por mejores condiciones ambientales (un ejemplo de ello es la cooperativa Los Milagros en Málaga). Por tanto, al ser la implementación en las barriadas obsoletas un proceso complejo, es muy posible que se produzca un riesgo de descapitalización social de sus residentes, al desplazarse sus activos más dinámicos hacia la periferia. Finalmente, hay que añadir que el uso de cooperativas de viviendas para facilitar el envejecimiento en el lugar, ha sido tratado en el ámbito rural (Lee, 2011), pero faltan aun investigaciones que analicen estas alternativas en el ámbito urbano, siendo las cooperativas vecinales una hipótesis de estudio que puede completar este gap.

\section{Metodología y caso de estudio}

Según la Encuesta Continua de Hogares de 2017 (INE, 2018), el 10,62\% de los hogares españoles está habitado por personas mayores que viven en soledad y que supone además el 41,83\% del total de hogares unipersonales. Una tendencia en aumento (en 2017 se produjo un incremento del 1,4\%) y que

\footnotetext{
3 Por ejemplo, los huertos urbanos del Parque de Miraflores de Sevilla (España), presentada en 1996 en la Conferencia Internacional HABITAT II de las Naciones Unidas son un ejemplo de buenas prácticas ciudadanas. Fueron dinamizadas por la población jubilada procedentes de las migraciones de los años 50 y 60.
}

ACE, 15 (4.5) CC BY-ND 3.0 ES | UPC Barcelona, España | Viabilidad económica de las cooperativas vecinales para 
afecta mayoritariamente a mujeres (1.410.000 hogares) frente a los hombres (550.900 hogares). En Andalucía, según datos del censo de 2001, más de 200.000 personas viven solas. En un análisis comparativo de las principales ciudades (Tabla 1), el porcentaje más alto se produce en Málaga y Sevilla.

Como caso de estudio del análisis comparativo, seleccionamos las barriadas de Sixto y Cortijo Vallejo en Málaga (España). Son barriadas que se localizan en la zona occidental de la ciudad, en el distrito de Carretera de Cádiz: una gran extensión de residencial construida principalmente durante los años 1960 y 1970, para absorber las migraciones del campo-ciudad. Desarrollado prácticamente en su totalidad con viviendas en bloques aislados de gran altura y con subvenciones oficiales. La barriada de Sixto se construyó a principios de 1960, en bloques de cinco plantas sin ascensor; mientras que la barriada de Cortijo Vallejo cuenta con 10 plantas y ascensor. Suman aproximadamente un total de 2.000 habitantes y en ambas barridas existen 114 viviendas en las que viven solas personas mayores de 65 años.

Tabla 1. Datos referentes al envejecimiento de las capitales andaluzas y a la oferta de viviendas en alquiler

\begin{tabular}{|c|c|c|c|c|c|c|c|c|c|}
\hline $\begin{array}{l}\text { Capital } \\
\text { andaluza }\end{array}$ & $\begin{array}{l}\text { Hab. } \\
(2017)\end{array}$ & $\begin{array}{l}>65 \\
\text { años } \\
\text { en hab. } \\
(2017)\end{array}$ & $\begin{array}{l}\% \text { de } \\
>65 \\
\text { años } \\
(2017)\end{array}$ & $\begin{array}{l}\text { № de } \\
\text { viv. } \\
\text { prin } \\
\text { (2011) }\end{array}$ & $\begin{array}{l}\text { № de } \\
\text { viv. } \\
\text { alquiler } \\
\text { (2015) }\end{array}$ & $\begin{array}{l}\text { № de } \\
\text { hogares } \\
\text { unipers. } \\
\text { mujer } \\
>65 \text { años } \\
(2001)\end{array}$ & $\begin{array}{l}\text { № de } \\
\text { hogares } \\
\text { unipers. } \\
\text { hombre } \\
>65 \text { años } \\
(2001)\end{array}$ & $\begin{array}{l}\text { № total } \\
\text { hogares } \\
\text { unipers. } \\
>65 \text { años } \\
(2001)\end{array}$ & $\begin{array}{l}\text { \% de } \\
\text { hogares } \\
\text { unipers } \\
>65 \\
\text { años } \\
(2001)\end{array}$ \\
\hline Sevilla & 689.434 & 130.165 & 18,88 & 268.435 & 42 & 34.472 & 9.536 & 44.008 & 33,81 \\
\hline Málaga & 569.002 & 96.787 & 17,01 & 211.358 & 859 & 27.106 & 9.497 & 36.603 & 37,82 \\
\hline Córdoba & 325.916 & 58.828 & 18,05 & 121.823 & 232 & 7.052 & 1.638 & 8.690 & 14,77 \\
\hline Granada & 232.770 & 48.626 & 20,89 & 96.916 & 97 & 7.234 & 1.424 & 8.658 & 17,81 \\
\hline Almería & 195.389 & 30.442 & 15,58 & 69.178 & 18 & 3.624 & 820 & 4.444 & 14,60 \\
\hline Huelva & 145.115 & 25.221 & 17,38 & 55.255 & 27 & 2.734 & 622 & 3.356 & 13,31 \\
\hline Cádiz & 118.048 & 26.667 & 22,59 & 46.966 & 83 & 3.003 & 704 & 3.707 & 13,90 \\
\hline Jaén & 114.238 & 19.580 & 17,14 & 43.258 & 43 & 2.496 & 526 & 3.022 & 15,43 \\
\hline Andalucía & - & - & - & 913.189 & - & 159.213 & 49.065 & 208.278 & 22,81 \\
\hline
\end{tabular}

Fuente: Elaboración propia a partir de datos del Instituto de Estadística y Cartografía de Andalucía, IECA (2019)

Para analizar el modelo teórico se hace un estudio empírico económico aproximativo de las ventajas de su implantación en una barriada de estudio. Para ello comparamos dos situaciones: una Situación $A$, referente a las personas mayores que viven en soledad en una barriada y afrontan la adaptación y mejora de las viviendas de forma autónoma e independiente; frente a una Situación B, en la que las personas mayores constituyen una CVPM para afrontar la misma adaptación. En ambas situaciones se hace un estudio de ingresos y gastos. Los datos de superficie y estado de la edificación de la barriada se obtienen del Instituto de Estadísticas y Cartografía de Andalucía (IECA, 2019). En los ingresos sólo se tienen en cuenta el alquiler de las viviendas liberadas, y para la estimación de los datos del mercado de alquiler se ha utilizado la plataforma electrónica del portal Fotocasa (2019). Mientras, respecto a los costes, se incluyen dos: 1) los costes de reforma de las viviendas, que se calculan con la estimación porcentual por capítulos para proyectos parciales y/o reformados según los costes de referencia del Colegio de Arquitectos de Málaga (COAM, 2019); y 2) los costes de la contratación del personal de apoyo.

En la Situación A, las obras de reforma corresponden normalmente a la adaptación de uno de los baños y a una mejora de las instalaciones. Mientras que, en la Situación $B$, el grado de intervención necesario determina que prioritariamente se deben modificar baños, cocinas y zonas de paso. Además, se requerirá la modificación de la compartimentación de dormitorios para adecuar sus dimensiones a la normativa de accesibilidad. Por ello, en la valoración del coste de reforma, se ha

ACE, 15 (4.5) CC BY-ND 3.0 ES | UPC Barcelona, España | Viabilidad económica de las cooperativas vecinales para 
tenido en cuenta la totalidad de la superficie construida. Las viviendas de la barriada de Sixto son viviendas mínimas que se pueden reformar para un programa de dos personas, con un baño adaptado, dos dormitorios de similares dimensiones y comedor-cocina. Mientras que las viviendas de Cortijo Vallejo, por lo general, son tipologías residenciales de cuatros dormitorios, adecuadas para adaptar un programa de tres personas mayores, mediante una redistribución interior que acoge un baño adaptado, tres dormitorios de similares dimensiones y comedor-cocina.

Según estos, se estima que la adaptación de locales a uso específico es un 50\% sobre el coste de referencia para el uso proyectado para calidades básicas equivalentes a viviendas de promoción oficial. El proyecto contempla que el servicio de mantenimiento asociado a cada inversión sea ofertado dentro del contrato de adjudicación de las acciones de mejora o la prestación del servicio. A diferencia del caso de las instalaciones, las soluciones constructivas para el interior de las viviendas se les exigen menos acciones de mantenimiento, lo que permite estimar su coste en un $10 \%$ del presupuesto de ejecución material.

\section{Resultados}

La constitución de una CVPM en las barriadas de estudio supone que para un conjunto de 114 viviendas de personas que viven solas, y de acuerdo con las características tipológicas de ambas barriadas, es posible agrupar socios en 46 viviendas adaptadas y liberar 68 viviendas en el mercado de alquiler (Tabla 2). Esto permite, por un lado, obtener unos ingresos estimados en $352.800 €$ anuales y, por otro, gestionar una oferta de viviendas de alquiler asequible con un precio de $400 €$ y $450 €$, unos precios muy por debajo de los precios de mercado existentes en la zona que rondan los $9 € / \mathrm{m}^{2}$ (Figura 3).

Tabla 2. Distribución de las viviendas de las personas mayores en la constitución de la cooperativa vecinal de personas mayores

\begin{tabular}{|l|l|l|l|l|l|l|}
\hline & \multicolumn{3}{|l|}{$\begin{array}{l}\text { Agrupación de personas mayores para evitar la } \\
\text { soledad }\end{array}$} & $\begin{array}{l}\text { Oferta de viviendas para alquiler de larga } \\
\text { estancia }\end{array}$ \\
\hline Barrida & $\begin{array}{l}\text { No viviendas } \\
\text { adaptadas }\end{array}$ & $\begin{array}{l}\text { № de socios } \\
\text { por vivienda } \\
\text { adaptada }\end{array}$ & $\begin{array}{l}\text { Total de } \\
\text { socios } \\
\text { agrupados }\end{array}$ & $\begin{array}{l}\text { No viviendas } \\
\text { liberadas } \\
\text { para alquiler }\end{array}$ & $\begin{array}{l}\text { Alquiler } \\
\text { mensual (€) }\end{array}$ & $\begin{array}{l}\text { Ingreso } \\
\text { anual (€) }\end{array}$ \\
\hline Sixto & 24 & 2 & 48 & 24 & 400 & 115.200 \\
\hline Cortijo Vallejo & 22 & 3 & 66 & 44 & 450 & 237.600 \\
\hline Total & 46 & - & 114 & 68 & 352.800 \\
\hline
\end{tabular}

Fuente: Elaboración propia.

Figura 3. Precio medio de alquiler en la zona de estudio de Málaga (España)

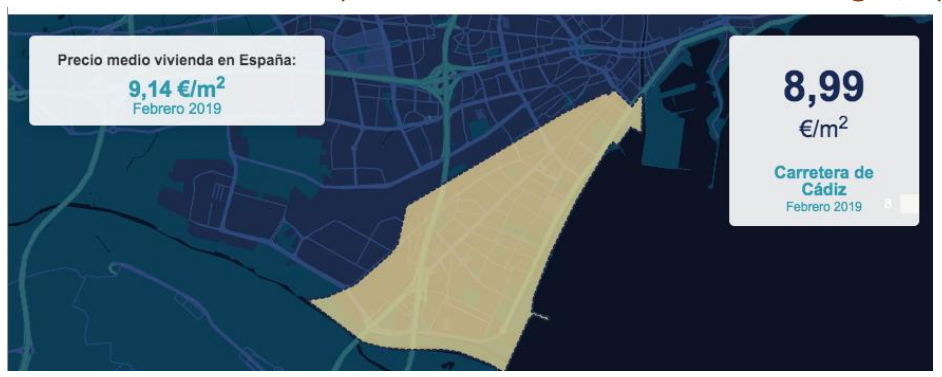

Fuente: Fotocasa (2019).

La agrupación de socios en viviendas compartidas permite reducir el número de viviendas a adaptar (Tabla 3), y llevar a cabo una reforma más integral que las que se llevan a cabo en muchos programas públicos, limitados a la simple adaptación de aseos o cuartos de baño.

ACE, 15 (4.5) CC BY-ND 3.0 ES | UPC Barcelona, España | Viabilidad económica de las cooperativas vecinales para el envejecimiento activo. Málaga como caso de estudio. DOI: http://dx.doi.org/10.5821/ace.15.45.10368 
Pero, además, la constitución de una CVPM permite aplicar una reducción del coste de construcción por economía de escala, ya que el volumen de obra a adaptar a los criterios del Código Técnico de la Edificación española y de las normas de accesibilidad universal de todas las viviendas de los socios, es muy superior al volumen de obra de cada propietario independiente, tal y como queda reflejado en la Tabla 4. Es decir, la rehabilitación conjunta de 46 viviendas es más económica que la rehabilitación individualizada de 46 viviendas. Dichos ahorros se resumen en tres:

a) Ahorro por eficiencia técnica. Al considerarse una intervención conjunta, el porcentaje de honorarios técnicos puede descender del 18\% al 11\% sobre el presupuesto de ejecución material, al poder aplicar un factor de repetición de las soluciones adoptadas.

b) Ahorro por eficiencia económica. Al contar con un volumen de obra importante, la CVPM puede beneficiarse de hasta una mejora económica del 25\% por oferta competitiva de la empresa que licite la obra, de forma similar a los procedimientos ordinarios de contratación pública.

c) Reducción en las tasas e impuestos municipales. El modelo asume la bonificación de las tasas e impuestos municipales al 100\% con base en el artículo 6 de la Ley de Ordenación Urbana de Andalucía, por el cual los ayuntamientos podrán aplicar bonificaciones sobre las tasas por expedición de licencias de obras para la conservación o rehabilitación de la edificación.

Tabla 3. Superficie construida de las viviendas adaptadas

\begin{tabular}{|l|l|r|r|}
\hline Barrida & № viv. & Sup. (m2) & Total sup. const. (m2) \\
\hline \hline Sixto & 18 & 65,00 & 1170,00 \\
& 6 & 55,00 & 330,00 \\
\hline Cortijo Vallejo & 6 & 85,00 & 510,00 \\
& 10 & 109,00 & 1090,00 \\
& 6 & 75,00 & 450,00 \\
\hline Total & 46 & - & 3550,00 \\
\hline
\end{tabular}

Fuente: Elaboración propia

Tabla 4. Costes de adaptación de las viviendas para cada una de las hipótesis de estudio

\begin{tabular}{|l|l|r|r|r|r|r|}
\hline Hipótesis & Agente & Base $(€)$ & Desc. $(\%)$ & Base reducida $(€)$ & IVA $(€)$ & Total $(€)$ \\
\hline \hline \multirow{3}{*}{$\begin{array}{l}\text { Sin cooperativa } \\
\text { Situación A }\end{array}$} & Contratista & 1.600 .000 & - & - & 160.000 & 1.760 .000 \\
\cline { 2 - 7 } & Proyectista & 216.000 & - & - & 45.000 & 261.000 \\
\cline { 2 - 7 } & Ayuntamiento & 78.100 & - & - & - & 78.000 \\
\cline { 2 - 7 } & Total & 1.845 .000 & - & - & 370.000 & 2.215 .000 \\
\hline \multirow{3}{*}{$\begin{array}{l}\text { Con cooperativa } \\
\text { Situación B }\end{array}$} & Contratista & 1.600 .000 & 25 & 1.200 .000 & - & 1.200 .000 \\
\cline { 2 - 7 } & Proyectista & 160.000 & 18 & 132.000 & - & 132.000 \\
\cline { 2 - 7 } & Ayuntamiento & 78.000 & 100 & - & - & - \\
\cline { 2 - 7 } & Total & 1.838 .000 & & 1.332 .000 & & 1.332 .000 \\
\hline
\end{tabular}

Fuente: Elaboración propia

Según se observa en la Tabla 4, para la misma obra de rehabilitación en un mismo número de viviendas, el ahorro en la situación B se ha estimado en $883.000 €$, ya que la CVPM no repercuta el IVA al ser una empresa, esto supone casi un 40\% de ahorro. Si suponemos un plazo de amortización de 10 años, el gasto de la rehabilitación para un interés muy reducido o subvencionado (1,5\%) se puede

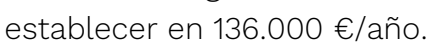

Por otro lado, la disponibilidad de ingresos permite la contratación de personal de apoyo a las personas mayores, se trataría de personal auxiliar que ayudaría en la limpieza de las viviendas y en el apoyo de las labores diarias (comida, compras, limpieza de ropa...). Con esto la CVPM permite la creación de puestos de trabajos de personal no cualificado que, además, puede llevar un seguimiento de las necesidades de las personas mayores. Como se observa en el resumen de ingresos y gastos

ACE, 15 (4.5) CC BY-ND 3.0 ES | UPC Barcelona, España | Viabilidad económica de las cooperativas vecinales para el envejecimiento activo. Málaga como caso de estudio. DOI: http://dx.doi.org/10.5821/ace.15.45.10368 
de la Tabla 5, los ingresos obtenidos por el alquiler de las viviendas liberadas permiten, además de financiar los gastos de rehabilitación, la contratación de 11 auxiliares de clínica con una ratio aproximada de 1 auxiliar por cada 10 socios.

Tabla 5. Viabilidad económica de la cooperativa para una amortización a 10 años

\begin{tabular}{|l|r|r|}
\hline Concepto & $\begin{array}{l}\text { Ingresos } \\
(€)\end{array}$ & $\begin{array}{l}\text { Gastos } \\
(€)\end{array}$ \\
\hline Alquiler de 68 viviendas & 352.800 & - \\
\hline $\begin{array}{l}\text { Rehabilitación y adaptación de } 46 \text { viviendas para agrupar las personas mayores en } \\
\text { unidades de } 203 \text { personas por vivienda (amortización a 10 años) }\end{array}$ & - & 136.000 \\
\hline Sueldo bruto anual de 10 auxiliares (18.000 €/año) & - & 180.000 \\
\hline Remanente de la CVPM & - & 36.800 \\
\hline Total & 352.800 & 352.800 \\
\hline Fuente: Elaboración propia
\end{tabular}

\section{Discusión y conclusiones}

La CVPM permite aunar el concepto del envejecimiento activo con el de envejecimiento en el lugar a través del cooperativismo. Por otra parte, el concepto de la CVPM apoya la agenda de investigación internacional de la ONU (y del Open-Ended Working Group on Ageing de la ONU) cuya reciente Resolución aprobada por la Asamblea General el 18 de diciembre de 2019 reconoce que "la planificación y creación de oportunidades para lograr que las ciudades incluyan la participación económica y social de las personas de edad es un aspecto importante de la construcción de ciudades sostenibles" (A/RES/74/125).

De acuerdo con los resultados obtenidos, la CVPM es un modelo de cooperativa que evita la soledad de las personas mayores de una barriada, agrupando en viviendas compartidas de dos o tres personas, bien en la propia vivienda o en viviendas cercanas, permitiendo con ello el envejecimiento en el lugar. Pero, a diferencia del modelo de co-vivienda, las personas mayores no precisan abandonar el barrio, ni requieren de un largo proceso de gestión hasta constituir la co-vivienda, ya que la CVPM sólo tiene que gestionar las viviendas existentes de los socios. Por otro lado, la adaptación de las viviendas a las necesidades de las personas mayores es más integral -superando las políticas que se limitan a la adaptación de baños o sustitución de bañeras-, ya que las viviendas son rehabilitadas y reformadas con nuevos programas habitacionales -más cercanos a las personas mayores-, e incorpora además prestaciones asistenciales de apoyo en la limpieza o comidas.

Desde el ámbito económico, la CVPM como empresa social, constituye un elemento de dinamización económica de la barriada, en consonancia con el modelo de envejecimiento positivo de Wiesel (2012), al aportar una fuente de financiación económica (el alquiler de viviendas) que, en el caso español, queda garantizado por la escasez de oferta de viviendas. Constituye, por tanto, una fuente complementaria de ingresos de las pensiones a partir de una economía y un beneficio sociales, ya que promueve una oferta de alquiler a precio tasado. El fomento de este modelo por parte de las administraciones públicas, puede ser una fórmula relativamente rápida de incrementar la oferta de vivienda de alquiler, previo al desarrollo de políticas de construcción de vivienda pública para el alquiler social. Si extrapolamos los resultados obtenidos, aproximadamente el 50\% de las personas mayores que viven solas pueden ofrecer su vivienda al alquiler de larga temporada que, en el caso de Málaga, puede representar una oferta de 3.600 viviendas de alquiler, y el de Andalucía hasta un total de 20.000 .

El modelo cuenta con limitaciones. Por un lado, es un modelo para personas mayores no dependientes y con autonomía, pero que deben de estar preparadas para asumir que comparten su vivienda con otras personas. Por otro lado, aunque las personas mayores siguen viviendo en el barrio, no todas lo hacen en su vivienda, por lo que puede haber resistencia a abandonar la misma. Además,

ACE, 15 (4.5) CC BY-ND 3.0 ES | UPC Barcelona, España | Viabilidad económica de las cooperativas vecinales para 
se produce una discriminación: las personas que rehabilitan sus viviendas se pueden sentir más beneficiadas que aquellas que ofrecen su vivienda en alquiler. Para futuras investigaciones, sería de especial interés, resolver estas cuestiones además de determinar el tamaño óptimo de la cooperativa en función de la capacidad de obtener recursos a partir del mercado de alquiler, la adaptación física de los espacios públicos y equipamientos urbanos de las barriadas para permitir un envejecimiento en el lugar; así como el desarrollo de redes sociales tecnológicas que faciliten la relación de los cooperativistas

\section{Agradecimientos}

Este artículo recoge los resultados del proyecto financiado por el Plan Propio de la Universidad de Málaga (PPIT.UMA.B1.2017/33). Asimismo, los autores quieren agradecer los comentarios y mejoras de los revisores de la revista que han evaluado el trabajo.

\section{Autoría}

El primer autor es responsable de la planificación del estudio, su redacción y análisis crítico. La segunda autora ha llevado a cabo la interpretación de resultados y el análisis crítico

Conflicto de intereses: Los autores declaran que no hay conflicto de intereses.

\section{Bibliografía}

Abellán, A.; Ayala, A. y Pujol, R. (2017). Un perfil de las personas mayores en España, 2017. Indicadores estadísticos básicos (Informes Envejecimiento en red № 15). Recuperado de http://envejecimiento.csic.es/documentos/documentos/enred-indicadoresbasicos17.pdf

Airbnb (2019). Recuperado de www.airbnb.es/.

Ayuso, M.; Guillén, M. y Valero, D. (2013). Sostenibilidad de las pensiones en España desde la perspectiva de la equidad y la eficiencia. Presupuesto y Gato Público, 71, 193-204.

Borgloh, S. \& Westerheide, P. (2012). The Impact of Mutual Support Based Housing Projects on the Costs of Care. Housing Studies, 27(5), 620-642. DOI: https://doi.org/10.1080/02673037.2012.697554

Brenton, M. (2008). The Cohousing Approach to Lifetime Neighbourhoods (Housing Learning \& Improvement Network facsheet no Recuperado 29). de https://www.housinglin.org.uk/ assets/Resources/Housing/Support materials/Factsheets/Factsheet 29.pdf

Choi, J. S. (2004). Evaluation of Community Planning and Life of Senior Cohousing Projects in Northern European Countries. European Planning Studies, 12(8), 1189-1216. DOI: https://doi.org/10.1080/0965431042000289296

COAM. (2019). Valores medios estimativos de la Construcción 2019. Recuperado de http://coamalaga.es/wp-content/uploads/2019/01/VALORES-MEDIOS-ESTIMATIVOS-DE-LACONSTRUCCIÓN-2019.pdf

ACE, 15 (4.5) CC BY-ND 3.0 ES | UPC Barcelona, España | Viabilidad económica de las cooperativas vecinales para el envejecimiento activo. Málaga como caso de estudio. DOI: http://dx.doi.org/10.5821/ace.15.45.10368 
Costa-Font, J.; Elvira, D., \& Mascarilla-Miró, O. (2009). "Ageing in place”? Exploring elderly people’s housing preferences in Spain. Urban Studies, 46(2), 295-316. DOI: https://doi.org/10.1177/0042098008099356

Decreto 28/2016, de 9 de diciembre, de las viviendas con fines turísticos y de modificación del Decreto 194/2010, de 20 de abril, de establecimientos de apartamentos turísticos. Boletín Oficial de la Junta de Andalucía, núm. 28, de 11 de febrero de 2016. Recuperado de https://www.juntadeandalucia.es/boja/2016/28/6

European Commission (2019). Peer Review on Strategies for supporting social inclusion at older age. Recuperado de https://ec.europa.eu/social/BlobServlet?docld=21809\&langld=en

European Commission Joint Research Centre. (2018). Loneliness - an unequally shared burden in Europe. Recuperado de https://ec.europa.eu/jrc/sites/jrcsh/files/fairness_pb2018 loneliness_jrc i1.pdf

Fernández Muñoz, J. N. (2015). Informe 2014. Las personas Mayores en España. Datos estadísticos estatales y por Comunidades Autónomas. Madrid: IMSERSO.

Fotocasa.

(2019).

Recuperado

de

https://www.fotocasa.es/indice/\#/filter/eyJpZCI6MTUwNSwidHlwZSI6NCwidHJhbnNhY3Rpb24iOiJyZ $\underline{W 50 \ln 0}=$.

Herce, J. A. (2016). El impacto del envejecimiento de la población en España. Cuadernos de Información Económica, 251, 39-48. Recuperado de http://www.ine.es/daco/daco42/sociales/infosoc_envej.pdf

Hui, E. C. M.; Wong, F. K. W.; Chung, K. W., \& Lau, K. Y. (2014). Housing affordability, preferences and expectations of elderly with government intervention. Habitat International, 43, 11-21. DOI: https://doi.org/10.1016/j.habitatint.2014.01.010

Idealista. (2019). Histórico de precios de alquiler en el Centro de Málaga. Recuperado de https://www.idealista.com/sala-de-prensa/informes-precio-vivienda/alquiler/andalucia/malagaprovincia/malaga/centro/historico/.

IECA. (2019). Instituto de Estadística y Cartografía de Andalucía. Recuperado de https://www.juntadeandalucia.es/institutodeestadisticaycartografia/temas/index-est.htm

INE. (2018). Encuesta Continua de Hogares. Año 2017. Notas de Prensa Del INE, pp. 2-11. Madrid. Recuperado de http://www.ine.es/prensa/np965.pdf

Lee, H.-J. (2011). Rediscovering a Path to Aging in Place: Development of Housing Cooperatives for Rural Elderly. Architectural Research, 13(3), 31-40. DOI: https://doi.org/10.5659/aikar.2011.13.3.31

López, J. (2005). Personas mayores viviendo solas. La autonomía como valor en alza. Madrid, España: IMSERSO.

López Rodríguez, D. y de los Llanos Matea, M. (2019). Evolución reciente del mercado de alquiler de vivienda en España. Artículos. Boletín Económico. Banco de España, 3. Recuperado de https://www.bde.es/f/webbde/SES/Secciones/Publicaciones/InformesBoletinesRevistas/ArticulosAn aliticos/19/T3/descargar/Fich/be1903-art25.pdf

ACE, 15 (4.5) CC BY-ND 3.0 ES | UPC Barcelona, España | Viabilidad económica de las cooperativas vecinales para el envejecimiento activo. Málaga como caso de estudio. DOI: http://dx.doi.org/10.5821/ace.15.45.10368 
Monzón, J. L. y Chaves, R. (2012). Evolución reciente de la economía social en la Unión Europea. Recuperado de https://www.eesc.europa.eu/sites/default/files/files/qe-04-17-875-es-n.pdf

Organización Mundial de la Salud. (2002). Envejecimiento activo: un marco político. Revista Española de Geriatría y Gerontología, 37(S2), 74-105. Recuperado de http://envejecimiento.csic.es/documentos/documentos/oms-envejecimiento-01.pdf

PEV (2018). Real Decreto 106/2018, de 9 de marzo, por el que se regula el Plan Estatal de Vivienda 2018-2021. Boletín Oficial del Estado, núm. 61, de 10 de marzo de 2018, pp. 28868-28916. Recuperado de https://www.boe.es/eli/es/rd/2018/03/09/106

Rosa-Jiménez, C., Márquez-Ballesteros, M., y Navas-Carrillo, D. (2017). Hacia un nuevo modelo de gestión y autofinanciación de la regeneración de barriadas obsoletas. Ciudades, 20, 45-70. DOI: https://doi.org/10.24197/ciudades.20.2017.26.

Sandercock, L. (2003). Towards Cosmopolis: Utopia as construction site. En S. S. Fainstein y J. Defilippis (Eds.), Readings in Planning Theory (pp. 401-407). Melbourne, Australia: Blackwell

Serrano-Jiménez, A., Barrios-Padura, Á. \& Molina-Huelva, M. (2018). Sustainable building renovation for an ageing population: Decision support system through an integral assessment method of architectural interventions. Sustainable Cities and Society, 39, 144-154. DOI: https://doi.org/10.1016/j.scs.2018.01.050

Wiesel, I. (2012). Can Ageing Improve Neighbourhoods? Revisiting Neighbourhood Life-Cycle Theory. Housing Theory Society, 29(2), 145-156. DOI: https://doi.org/10.1080/14036096.2011.641258

Zuribi, I. (2003). El futuro del sistema de pensiones en España. Madrid, España: Instituto de Estudios Fiscales.

ACE, 15 (4.5) CC BY-ND 3.0 ES | UPC Barcelona, España | Viabilidad económica de las cooperativas vecinales para el envejecimiento activo. Málaga como caso de estudio. DOI: http://dx.doi.org/10.5821/ace.15.45.10368 